\title{
Life-time and pinning effects in CDW systems
}

\author{
W. AICHMANN and I. BÂLDEA $(1)$ \\ Universität Ulm, Abteilung für Mathematische Physik, Oberer Eselsberg, Postfach 4066, \\ 89069 Ulm/Donau, Germany
}

\begin{abstract}
We develop here a microscopical formalism enabling the simultaneous treatment of the two main effects due to electron-impurity interaction: finite electronic life-time and pinning of the CDW phase. It is found that these two effects affect each other in a way which is particularly non-trivial in the strong pinning limit.
\end{abstract}

\section{Introduction.}

The disturbance caused by immersing non-magnetic impurities into the CDW system is mainly two-fold: ( $i$ ) the destruction of the CDW properties via the life-time effect and (ii) the pinning of the CDW phase. Essentially, the previous studies have been concentrated on either the one or the other of the these phenomena. It is the aim of the present work to investigate both effects on equal footing within the self-consistent single-site approximation. We thus improve the approximation employed in a previous study (Bâldea, unpublished), where the electron-impurity scattering has been treated within the self-consistent Born approximation; there, the analytic expressions for the relevant quantities have been obtained.

By restricting ourselves to electron-impurity backscattering processes, there are two kinds of terms in the present approach. Firstly, those whose wave vectors $q$ are equal to the CDW wave vector $Q$; they are the terms responsible for the pinning of the CDW phase, retained by the conventional Fukuyama-Lee approach [1]. Secondly, those with $q \approx Q(q \neq Q)$ associated with the incoherent scattering of electrons by randomly distributed impurities which bring about the finite width of single-electron levels (e.g. [2] and references cited therein). The main technical aspect of the present calculations is the the fact that we use the same procedure of averaging for both. The employed averaging procedure straightforwardly yields several terms in the electron self-energy which are nonvanishing only in the presence of pinning; consequently, they are specific to the present work, which accounts simultaneously for CDW pinning and finite electronic life-time.

\section{Formalism.}

Following the standard method (e. g. $[3,4]$ ), the Hamiltonian of the one-dimensional (1D) CDW state in the presence of non-magnetic impurities can be cast into the form:

$$
H=H_{C D W}^{0}+H_{i m p, 1}+H_{\theta}
$$




$$
\begin{aligned}
H_{C D W}^{0} & =\int d x \hat{\Psi}^{\dagger}(x)\left[-i v_{F} \hat{\tau}_{3} \frac{\partial}{\partial x}+\left(\Delta+V_{B, 0}\right) \hat{\tau}_{+}+\left(\Delta+V_{B, 0}^{*}\right) \hat{\tau}_{-}\right] \hat{\Psi}(x)+\frac{L}{\pi \lambda v_{F}} \Delta^{2} \\
H_{i m p, 1} & =\int d x \hat{\Psi}^{\dagger}(x)\left[V_{B, 1}(x) \hat{\tau}_{+}+V_{B, 1}^{*}(x) \hat{\tau}_{-}\right] \hat{\Psi}(x) \\
H_{\theta} & \equiv H_{\theta 1}+H_{\theta 2}=\int d x \hat{\Psi}^{\dagger}(x)\left[\frac{v_{F}}{2} \frac{\partial \theta}{\partial x}+\frac{1}{2 m}\left(\frac{\partial \theta}{\partial x}\right)^{2}\right] \hat{\Psi}(x) \\
V_{B, 0} & =L^{-1} V_{e-i}(Q) \sum_{\alpha=1}^{N_{i}} s_{\alpha} \\
V_{B, 1}(x) & =L^{-1} V_{e-i}(Q) \sum_{\alpha=1}^{N_{i}} \sum_{q \neq 0} \exp \left[-i q\left(x-Y_{\alpha}\right)\right] s_{\alpha} \\
s_{\alpha} & \equiv \exp \left[-i\left(Q Y_{\alpha}+\theta_{\alpha}\right)\right] .
\end{aligned}
$$

Here, $\Psi$ and $\Psi^{\dagger}$ are, as usual, the Nambu spinors, $\Delta$ is amplitude of the lattice distorted field, $\hat{\tau}_{ \pm}=\left(\hat{\tau}_{+} \pm i \hat{\tau}_{-}\right) / 2$ ( $\hat{\tau}^{\prime}$ s - Pauli matrices in band space), $\lambda$ and $v_{F}$ stand for the electron-phonon coupling strength and Fermi velocity, $m$ and $L$ denote the band electronic mass and the length of the system, respectively. Only backscattering components of the electron-impurity interaction $V_{e-i}$ are included above and, furthermore, their $q$-dependence is ignored. The latter (amounting to the assumption of a contact interaction) yields the presence of the parameters $\left\{\theta_{\alpha}\right\}$ (values of the CDW phase at the positions $\left\{Y_{\alpha}\right\}$ ) of the $N_{i}$ impurity atoms (ions)).

One should note the splitting of the electron-impurity interaction in terms with $q=0$ and $q \neq 0$, which leads to different effects: CDW pinning and finite electronic life-time, respectively. Noteworthy is the presence of the same phase factors $s_{\alpha}$ in both Eqs. (5) and (6). As mentioned in Introduction, the previous studies devoted to the CDW pinning have usually ignored the term $H_{i m p, 1}$, while those devoted to the life-time effect assumed $V_{B, 0}=0$ and a rigid CDW phase (i. e. $\theta_{\alpha}=\theta=$ const) in Eq. (6). The variational solution is obtained by minimizing the thermodynamical potential with respect to $\Delta$ and $\left\{\theta_{\alpha}\right\}$.

The contribution of $V_{B, 0}$ can be exactly computed, by diagonalizing $H_{C D W}^{0}$ (Eq. (2)); while its lowest order contribution gives the potential energy in the treatment by Fukuyama-Lee [1], its general effect is to enhance the distortion field from the value $\Delta$ in the non-pinned situation to:

$$
\Delta_{M F^{\prime}}=\Delta+V_{B, 0} .
$$

This renormalization effect has already been pointed out [4].

The term $H_{i m p, 1}$ (Eq. (3)), yielding the finite life-time of electron levels, will be treated here within the self-consistent single-site approximation (e. g. [5]). One thus gets the following expression for the electron Green function ( $\epsilon$ - Matsubara frequencies):

$$
\hat{G}(p, i \epsilon)=-\frac{i \tilde{\epsilon} \hat{\tau}_{0}+v_{F} p \hat{\tau}_{3}+\tilde{\Delta} \hat{\tau}_{+}+\tilde{\Delta}^{*} \hat{\tau}_{-}}{\tilde{\epsilon}^{2}+\left(v_{F} p\right)^{2}+|\tilde{\Delta}|^{2}},
$$

where $\left(U_{B} \equiv\left|V_{e-i}(Q)\right|\right)$ :

$$
\begin{aligned}
& \tilde{\epsilon}=\epsilon+\frac{U_{B}^{2}}{2 v_{F}} \frac{\tilde{\epsilon}}{\sqrt{\tilde{\epsilon}^{2}+|\tilde{\Delta}|^{2}}} \frac{1}{L} \sum_{\alpha} \frac{1}{1+\left(\frac{U_{B}}{2 v_{F}}\right)^{2}+\frac{U_{B}}{2 v_{F}} \frac{\tilde{\Delta} s_{\alpha}^{*}+\tilde{\Delta}^{*} s_{\alpha}}{\sqrt{\tilde{\epsilon}^{2}+|\tilde{\Delta}|^{2}}}}, \\
& \tilde{\Delta}=\Delta_{M F}+\frac{U_{B}}{L} \sum_{\alpha}\left\{\frac{\frac{U_{B}}{2 v_{F}} \frac{\tilde{\Delta}}{\sqrt{\tilde{\epsilon}^{2}+|\tilde{\Delta}|^{2}}}+s_{\alpha}}{\left.1+\left(\frac{U_{B}}{2 v_{F}}\right)^{2}+\frac{U_{B}}{2 v_{F}} \frac{\tilde{\Delta} s_{\alpha}^{*}+\tilde{\Delta}^{*} s_{\alpha}}{\sqrt{\tilde{\epsilon}^{2}+|\tilde{\Delta}|^{2}}}-s_{\alpha}\right\} .} .\right.
\end{aligned}
$$

In the absence of pinning the summations over $\alpha$ in Eqs. (10) and (11) containing (powers of) the phase factors $\left\{s_{\alpha}\right\}$ (Eq. (7)) give no contribution; then the second-order expansion in $U_{B}$ recovers 
the results obtained within the Born approximation (e. g. [2,6]). However, they give a non-vanishing contribution for the pinned CDW. This shows that the pinning affects the CDW order parameter both directly (cf. Eq. (8)) and indirectly (via the alteration of the life-time effect, cf. Eqs. (10) and (11)).

By treating the term $H_{\theta, 1}$ in the second order of perturbation theory, the contribution of $H_{\theta}$ (Eq. (4)) is an elastic energy which has to be paid to pin the CDW phase. By extending the method employed in the absence of life-time effect [4] such that to include both self-energy and vertex corrections caused by $H_{i m p, 1}$ [2] it can be obtained in the form:

$$
E_{\text {elastic, } \theta}=L \frac{v_{F}}{4 \pi}\left\langle\frac{\partial \theta}{\partial x}\right\rangle^{2}\left(1-\frac{\chi}{\chi_{N}}\right),
$$

$\left(\chi / \chi_{N}\right.$ - static spin susceptibility normalized to the value of the normal $1 D$ metal [2]) which differs from the Fukuyama-Lee expression [1] by the factor in the last paranthesis, incorporating the temperature effect (a fact pointed out previously [4]) as well as the electronic life-time effect.

The full consequences of the preceding results will be not discussed here. However, even the presentation of the results for strong pinning case in the dilute limit at $T=0$, to which we restrict ourselves below, could reveal that these consequences are non-trivial. The linear expansion in the impurity concentration $n_{i}\left(\equiv N_{i} / L\right)$ yields the following expressions for $\Delta$ in the non-pinned and strongly-pinned situations (specified by the indices $n$ and s.p., respectively $(\alpha=n, s . p$.$) ):$

$$
\begin{aligned}
\Delta_{\alpha} & =\Delta_{0}+2 v_{F} n_{i} S_{\delta, \alpha} \\
S_{\delta, n} & =-\eta^{2} \int_{0}^{\infty} d x \frac{x^{2}\left(1+x^{2}\right)^{-3 / 2}}{\sqrt{1+x^{2}-4 \eta^{2}}}\left\{1+\frac{2 \eta^{2}}{1+x^{2}-2 \eta^{2}+\sqrt{1+x^{2}} \sqrt{1+x^{2}-4 \eta^{2}}}\right\}, \\
S_{\delta, s, p .} & =\frac{1}{\lambda} \frac{u}{1+u^{2}}+\frac{u^{2}-1}{u^{2}+1} \arctan \frac{u-1}{u+1},
\end{aligned}
$$

where $u \equiv U_{B} / 2 v_{F}$ and $\eta \equiv 1 /(u+1 / u)$. Noteworthy, the increase in the order parameter $\Delta$ in
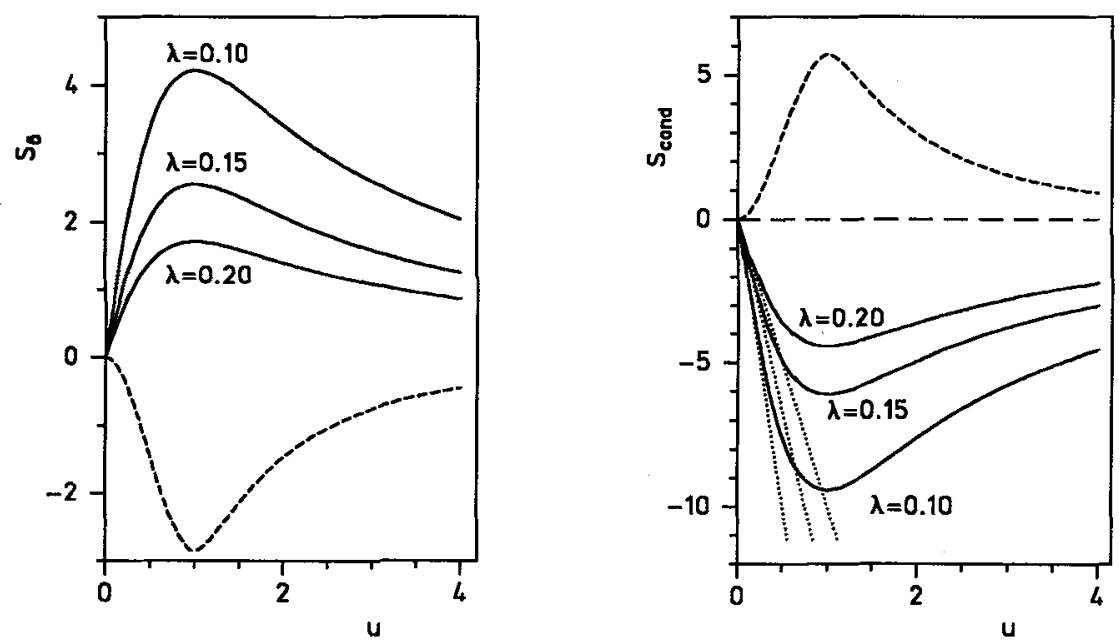

Figure 1: Dependence of the slope parameters $S_{\delta}$ (left) and $S_{\text {cond }}$ (right) (Eqs. (13) and (14)) on the dimensionless scattering potential $u \equiv U_{B} / 2 v_{F}$ for non-pinned (dashed line) and strongly pinned (solid lines) CDW. The dotted lines represent (tangent to the solid ones) result from the FukuyamaLee calculations [1]. See the main text for details.

the pinned situation by increasing $n_{i}$, in contrast to its decrease (due to the life-time effect) in the absence of pinning. This is illustrated in Fig. 1 by the different signs of the parameters $S_{\delta, n}$ and 
$S_{6, \text { s.p. }}$, where, furthermore, their dependences on $u$ is visualized. Within the same approximations, the expressions of the condensation energy (divided by the metallic density of states) read:

$$
\begin{aligned}
\epsilon_{\text {cond, } \alpha} & =-\frac{\Delta_{0}^{2}}{2}-4 v_{F} \Delta_{0} S_{\text {cond }, \alpha} n_{i}, \\
S_{\text {cond, } n} & =S_{\delta, n}, \\
S_{\text {cond,s.p. }} & =S_{\delta, \text { s.p. }}+\eta .
\end{aligned}
$$

In the lowest order in $u$, the correction term in $\epsilon_{\text {cond, s.p. }}$ expressed above reduces to that obtained in the Fukuyama-Lee approach (for 1D), $-2 U_{B} \Delta_{0} n_{i} / \lambda[1]$. The dependence of $S_{\text {cond }}$ on $u$ is also displayed in Fig. 1.

\section{Conclusion.}

The simultaneous inclusion of both afore-mentioned effects is justified by the fact that their mutual influence is non-trivial. The CDW order parameter is renormalized both by the incoherent electronimpurity scattering and by the pinning of the CDW phase. On the other side, the electronic life-time is modified in the case of pinned CDW phase as compared to the non-pinned situation: for instance, this can be seen in the different contributions to the condensation energy. The change in the condensation energy should reflect itself in the threshold field for depinning.

More detailed results (obtained both within the self-consistent Born and single-site approximations) will be reported elsewhere.

\section{Acknowledgements.}

One of the authors (I. B.) would like to thank the financial support provided by the Alexander von Humboldt Foundation during the main part of this work and by the German Academic Exchange Service (D A A D) in its initial stage as well as the hospitality extended to him at the University of Ulm. Valuable discussions with Prof. Wolfgang Wonneberger are gratefully acknowledged.

\section{References}

[1] Fukuyama H. and Lee P. A., Phys. Rev. B 17 (1978) 535; Lee P. A. and Fukuyama H., Phys. Rev. B 17 (1978) 542.

[2] Bâldea I., Physica Scripta 42 (1990) 749 and references cited therein.

[3] Brazovskii S. and Dzhyaloshinskii I. E., Sov. Phys.-J E T P 44 (1976) 1233.

[4] Nakane Y. and Takada S., J. Phys. Soc. Japan 57 (1988) 217; 4297.

[5] Elliot R. J., Krumhansl J. A. and Leath P. L., Rev. Mod. Phys. 46 (1974) 465.

[6] Maki K., in Superconductivity Ed. D. Parks, vol, 2, p. 1035 (Dekker, New York, 1969). 